\title{
A blind passenger: a rare case of documented seroconversion in an Angiostrongylus cantonensis induced eosinophilic meningitis in a traveler visiting friends and relatives
}

\author{
Tobias Brummaier ${ }^{1,2,3,4^{*}}$ (D), Sonja Bertschy ${ }^{5}$, Kornelius Arn ${ }^{6}$, Thomas Treumann ${ }^{7}$, Marie-Therese Ruf ${ }^{1,2}$, \\ Beatrice Nickel ${ }^{1,2}$, Daniel H. Paris ${ }^{1,2}$, Andreas Neumayr ${ }^{1,2}$ and Johannes Blum ${ }^{1,2}$
}

\begin{abstract}
Background: Eosinophilic meningitis (EOM) is a rare condition that is caused by various communicable and noncommunicable factors. The rat-lungworm Angiostrongylus cantonensis, which is associated with consumption of raw or undercooked paratenic or intermediate hosts, is the most common cause of parasitic eosinophilic meningitis worldwide. While the majority of $A$. cantonensis cases are reported from endemic regions, cases in travelers pose a challenge to clinicians in non-endemic countries. Here we report a rare case of eosinophilic meningitis caused by $A$. cantonensis in a Swiss traveler who was diagnosed after returning from Thailand.

Case presentation: A 33-year old woman with a travel history to rural north-eastern Thailand presented to an emergency department in Switzerland with severe headache and vomiting. Eosinophilic meningitis was confirmed as the cause of the symptoms; however, serologic investigations failed to confirm an A. cantonensis infection on the first evaluation. Nevertheless, empirical treatment with an anthelminthic and steroid regimen led to a rapid alleviation of symptoms. Repeated serology confirmed seroconversion 2 weeks after treatment initiation.

Discussion: Parasitic etiology must be considered in returning travelers who present with symptoms compatible with a central nervous system infection. A thorough medical history, including types of food consumed, is paramount and can often suggest differential diagnosis. Neuroangiostrongyliasis is rare and might be missed if serology does not cover possible seroconversion.
\end{abstract}

Keywords: Angiostrongylus cantonensis, Eosinophilic meningitis, Seroconversion, Switzerland, Visiting friends and relatives

\section{Background}

Parasitic central nervous system (CNS) infections are expected to become more common in non-endemic countries. Parasitic etiologies require more consideration in travelers presenting with symptoms suggestive of a CNS infection. Overlapping and often non-specific clinical symptoms in combination with their scarcity and epidemiologic

\footnotetext{
* Correspondence: tobias.brummaier@gmx.at

'Department of Medicine, Swiss Tropical and Public Health Institute, Basel, Switzerland

${ }^{2}$ Faculty of Medicine, University of Basel, Basel, Switzerland

Full list of author information is available at the end of the article
}

peculiarities make parasitic CNS infections a diagnostic challenge [1]. The rat-lungworm Angiostrongylus cantonensis is the most common cause of parasitic eosinophilic meningitis (EOM) worldwide [2]. Infective third-stage Angiostrongylus larvae are found in intermediate hosts (i.e. gastropods such as snails or slugs), food products contaminated with gastropods or gastropod mucus (e.g. vegetables, fruits) or in paratenic hosts (e.g. shrimp, prawns, crabs, monitor lizards, frogs, mussels). Accidental or deliberate ingestion of raw or undercooked food products containing the neurotropic third-stage larvae causes CNS infections in humans [3]. Larvae pass the gastrointestinal tract, actively

(c) The Author(s). 2019 Open Access This article is distributed under the terms of the Creative Commons Attribution 4.0 International License (http://creativecommons.org/licenses/by/4.0/), which permits unrestricted use, distribution, and 
cross into systemic circulation and migrate to the central nervous system. Symptoms occur after an incubation period that varies from 7 to 35 days [3].

A. cantonensis cases are primarily reported from endemic regions (Fig. 1) [4]. However, due to an increase in international travel, individual tourism, and a change in risk behavior, an increment in sporadic cases of $A$. cantonensis in non-endemic countries is expected [5]. The rare occurrence and usually self-limiting course of the disease are 2 factors that contribute to the assumption that many cases of $A$. cantonensis remain undiagnosed.

Here we present a rare case of an EOM caused by $A$. cantonensis in a visiting friends and relatives (VFR) traveler.

\section{The case}

In August 2018 a 33-year old woman presented with a history of progressive headache, nausea and vomiting. The patient is of Thai origin but has resided in Switzerland for 6 years. However, she regularly travels to rural north-eastern Thailand, which was also the case prior to onset of symptoms. Two weeks after arrival in Thailand, the patient noted self-limiting diarrhea that was followed by non-specific symptoms (i.e. fatigue, feeling of generalized swelling, leg accentuated weakness).
Two weeks after onset of these non-specific symptoms the patient returned to Switzerland and reported symptom aggravation in the form of gradually increasing headache. The initial headache location shifted from the right to the left hemisphere and eventually manifested as holocranial headache of dull-throbbing character. The patient, who denied being prone to headaches, described severe headache (numerical rating scale $7-10$ ) that was not responsive to nonsteroidal anti-inflammatory drugs. Insomnia due to persistence of headache with additional occurrence of nausea and vomiting prompted the patient to attend the emergency department 5 days after returning from Thailand.

On admission, the patient was afebrile (temperature $36,8^{\circ} \mathrm{C}$ ), fully conscious (Glasgow Coma Scale 15) and without focal neurologic deficits or signs of meningism. The medical history revealed a pre-existing, inactive hepatitis B infection and subclinical hypothyroidism. For both conditions no treatment was taken at the time of presentation. From routine screening during a previous pregnancy, HIV was known the be negative. A complete blood count $(\mathrm{CBC})$, clinical chemistry, a lumbar puncture (LP) and cranial computed tomography (CT) was ordered. The $\mathrm{CBC}$ indicated a neutrophil predominance and biochemistry results were unremarkable. In the

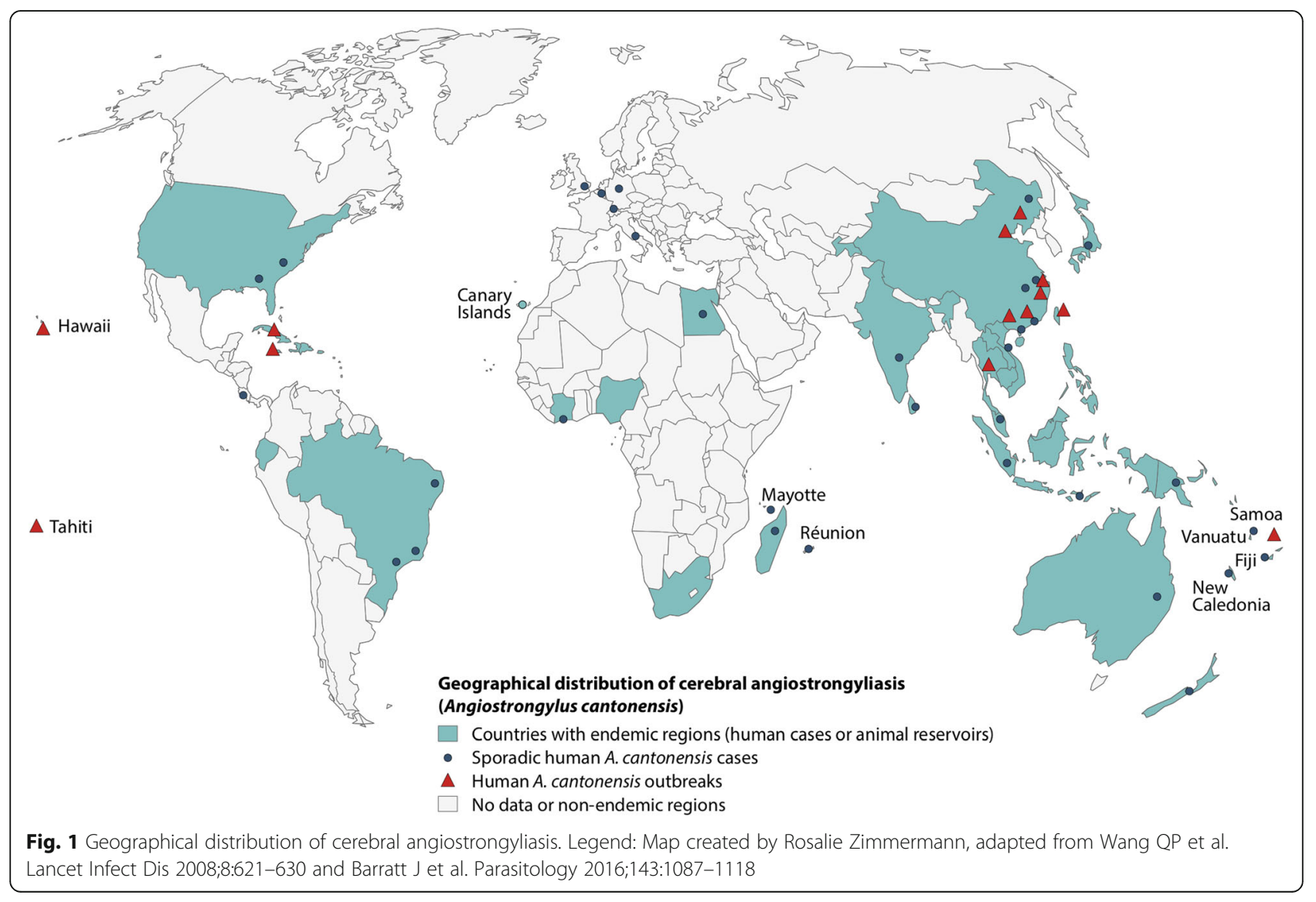


cerebrospinal fluid (CSF) eosinophilia was noted (Table 1), a finding that was not reflected in the peripheral differential count.

A vascular occlusion in the supply area of the right middle cerebral artery compatible with Moyamoya disease was suspected in the CT scan. Magnetic resonance imaging (MRI) was performed, revealing adequate collateral circulation and no evidence for a recent occlusion or active vasculitis. A few age-appropriate non-specific white-matter lesions were seen in both hemispheres but no leptomeningeal nor nodular enhancing lesions.

The pleocytosis (cell count of $1067 / \mu \mathrm{L}$ ) in the CSF, and the abnormally elevated absolute and relative eosinophil counts of $267 / \mu \mathrm{L}$ and $25 \%$ respectively, led to the diagnosis of EOM (Fig. 2). Considering the travel history, an empirical treatment with albendazole (400 $\mathrm{mg}, \mathrm{BD}$ ) and prednisolone (60 $\mathrm{mg} \mathrm{OD}$ ) was started to cover parasitic EOM. Additionally, serological testing for tissue helminths was ordered and performed at the

Table 1 Cerebrospinal fluid and blood results

\begin{tabular}{|c|c|c|}
\hline Test (Unit) & Result & Reference Range \\
\hline \multicolumn{3}{|l|}{ Cerebrospinal fluid } \\
\hline - Appearance & Cloudy & Clear \\
\hline - Xanthochromia & Negative & Negative \\
\hline - Erythrocytes $\left(\times 10^{12} / \mathrm{L}\right)$ & 0 & 0 \\
\hline - Cell count $\left(\times 10^{9} / \mathrm{L}\right)$ & 1067 & $0-3$ \\
\hline • Lymphocytes (\%) & 56 & \\
\hline - Monocytes (\%) & 19 & \\
\hline • Eosinophils (\%) & 25 & $\neq$ \\
\hline • Total protein (g/L) & 0.54 & $0.15-0.45$ \\
\hline • Albumin (g/L) & 0.299 & $0.06-0.24$ \\
\hline - Glucose (mmol/L) & 2.6 & $2.2-3.9$ \\
\hline - Lactate (mmol/L) & 1.8 & $1.2-3.9$ \\
\hline \multicolumn{3}{|l|}{ Complete Blood Count } \\
\hline - Leukocytes $\left(\times 10^{9} / \mathrm{L}\right)$ & 6.5 & $2.6-7.8$ \\
\hline o Neutrophils $\left(\times 10^{9} / \mathrm{L}\right)$ & 6.21 & $0.9-4.5$ \\
\hline o Banded neutrophils $\left(\times 10^{9} / \mathrm{L}\right)$ & 0.04 & $0.0-0.2$ \\
\hline o Eosinophils $\left(\times 10^{9} / \mathrm{L}\right)$ & 0.01 & $0.0-0.4$ \\
\hline o Basophils $\left(\times 10^{9} / \mathrm{L}\right)$ & 0.01 & $0.0-0.05$ \\
\hline o Monocytes $\left(\times 10^{9} / \mathrm{L}\right)$ & 0.05 & $0.0-1.0$ \\
\hline o Lymphocytes (×109/L) & 0.79 & $1.0-3.0$ \\
\hline - Erythrocytes $\left(\times 10^{12} / \mathrm{L}\right)$ & 4.16 & $3.7-5.0$ \\
\hline • Hemoglobin (g/L) & 117 & $115-148$ \\
\hline - Hematocrit & 0.35 & $0.34-0.43$ \\
\hline - Platelet $\left(\times 10^{9} / \mathrm{L}\right)$ & 193 & $130-330$ \\
\hline - $\mathrm{C}$ reactive protein $(\mathrm{mg} / \mathrm{L})$ & $<5$ & $<5$ \\
\hline
\end{tabular}

Abnormal results are shown in bold

${ }^{\text {a }}$ Reference ranges according to the hospital laboratory

\#Eosinophilic meningitis is defined as 10 or more eosinophils $/ \mu \mathrm{L}$ or

eosinophilia of at least $10 \%$ of the total CSF leukocyte count [8]

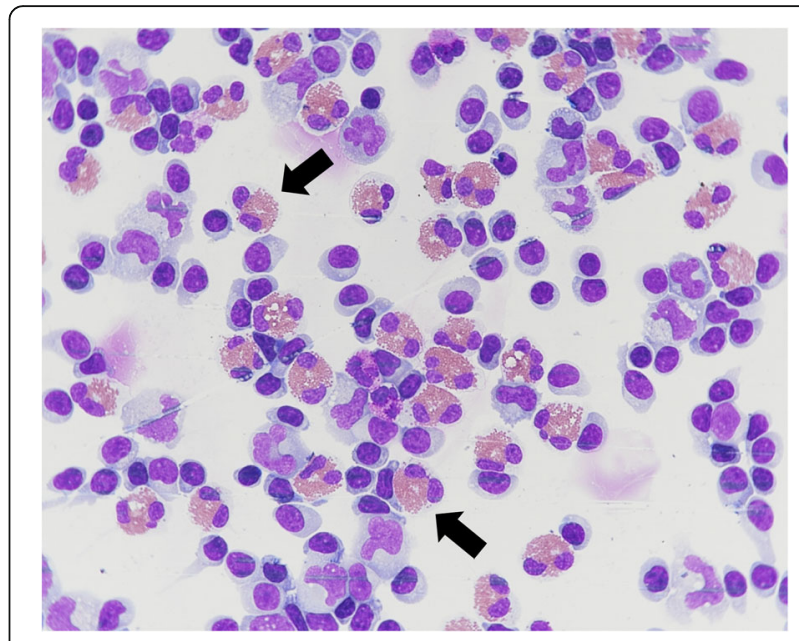

Fig. 2 Cerebrospinal fluid smear of our patient. Legend: Pappenheim (May-Grünwald-Giemsa) stain, magnification $\times 60$. Black arrows indicate eosinophil granulocytes with mostly bilobed, occasionally trilobed nucleus and normal eosinophilic granulation

national reference center at Swiss Tropical and Public Health Institute, Basel, Switzerland alongside other neurotropic pathogens.

Enzyme-linked electroimmunotransfer blot (EITB) for A. cantonensis and Gnathostoma spp specific IgG antibodies performed with serum were nonreactive. IgG screening ELISAs and IgG/IgM/IgA indirect fluorescent antibody tests for Echinococcus granulosus, Fasciola spp., Filaria spp. Schistosoma spp., Strongyloides stercoralis, Trichinella spp and Toxocara spp. as well as screening for other neurotropic pathogens (i.e. Borrelia burgdorferi, Tick-borne encephalitis virus, Treponema pallidum) and HIV were negative. Aside from the afore-mentioned pathogens, the origin of symptoms due to an infection with free living amoeba (FLA) was considered. However, since neither typical features of primary amoebic meningoencephalitis (caused by Naegleria) nor granulomatous amoebic encephalitis (caused by Acanthamoeba) were seen, no molecular or immunologic tests to rule out a FLA infection were requested.

As acute phase Angiostrongylus serology can be negative, the empirical treatment was continued and follow up serology was recommended. The dual anthelminthic and anti-inflammatory therapy led to a marked clinical improvement, and the patient was discharged home after 6 days of hospitalization.

Two weeks after treatment initiation, the patient was seen for a follow up visit in the outpatient department. At this visit, no more symptoms were reported and serologic testing for A. cantonensis and Gnathostoma spp, the two most likely causes of the initial symptoms were repeated. While the latter remained negative, the serological test for $A$. cantonensis turned positive, indicating 
seroconversion and, thus confirming the Angiostrongylu$s$-associated EOM. Recommended treatment with albendazole (3 weeks) and prednisolone (2 weeks) was completed and the patient recovered completely.

\section{Discussion}

Human health and travelers' health in particular is strongly influenced by altering interrelationships between international travel, globalization of trade and agriculture, migration as well as food production in a "warming world" [6]. In addition, ripple effects of adventure travel, exotic eating habits and exotic pet trade have repercussions on the epidemiology of communicable diseases that are traditionally considered tropical diseases [6].

In parasitic CNS infections, signs and symptoms are often non-specific and/or overlapping making clinical diagnosis arduous. Travelers returning from potentially endemic countries complaining about severe headache should be generously evaluated for parasitic CNS infections. A thorough travel history and assessment of risk behavior while traveling can guide diagnosis and direct further diagnostic measures. This patient ingested uncooked freshwater mussels from a lake in rural north-eastern Thailand 20 days before the headache commenced.
Magnetic resonance imaging is the radiologic method of choice if parasitic CNS infections are suspected [7]. However, findings in neuroimaging studies have their limitations and also did not contribute significantly to the confirmation of the diagnosis in this patient.

Confirmatory diagnosis is based on laboratory methods [1]. CSF investigations are paramount in the workup if meningitis is suspected to confirm EOM (i.e. an eosinophil count of $\geq 10$ eosinophils per microliter, or eosinophilia of $\geq 10 \%$ of the total CSF leukocyte count [8]). Various parasitic causes of EOM have to be considered and ruled out where applicable (Fig. 3). CSF xanthochromia is an indicator for the distinction between $A$. cantonensis and Gnathostomiasis as it is suggestive of the latter [9]. Absent xanthochromia led to presumptive diagnosis of A. cantonensis in the presented case and guided the decision-making process for empirical treatment.

Most cases manifest symptoms in the first 1-2 weeks after larva ingestion. However, incubation periods of months [10] and autochthonous A. cantonensis cases from non-endemic areas have been reported [11, 12]. EOM or meningoencephalitis are the most common manifestations followed by ocular angiostrongyliasis [5, 13]. The cardinal symptom in EOM is headache and affects up to $95 \%$ of cases [3]. Vasodilatation in the subarachnoid space resulting

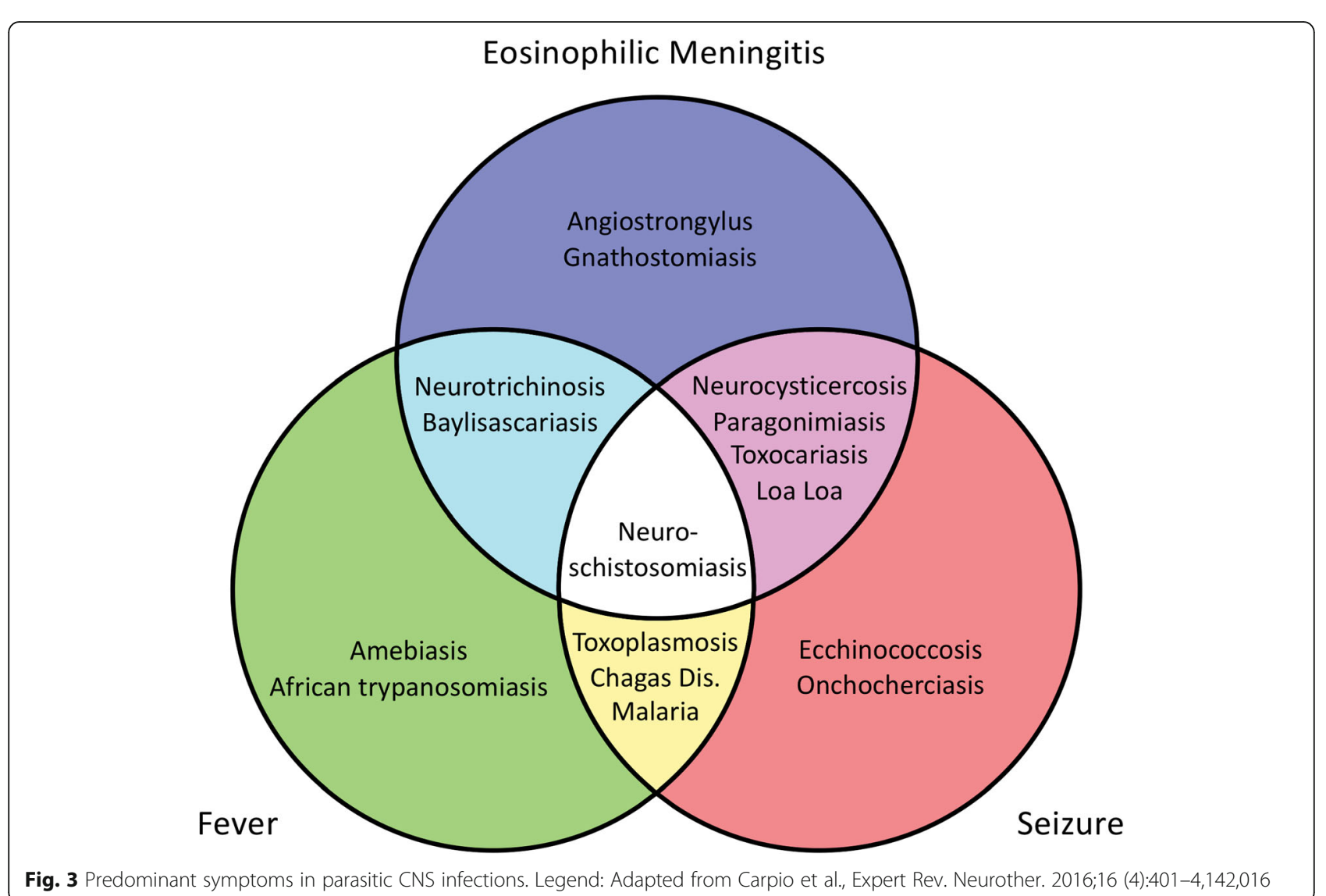

Fig. 3 Predominant symptoms in parasitic CNS infections. Legend: Adapted from Carpio et al., Expert Rev. Neurother. 2016;16 (4):401-4,142,016 
from widespread inflammatory reaction in the meninges and decreased resorption of CSF lead to increased intracranial pressure, explaining other neurologic findings (e.g. neck stiffness, paresthesia, cerebral nerve palsy, nausea and vomiting) associated with neuroangiostrongyliasis [9, 14].

Since larva recovery from CSF is rare, confirmation is mainly based on detection of antibodies in serum or CSF. Unfortunately, there was not enough residual CSF to perform serologic tests in the patient presented here. Detection of $A$. cantonensis DNA by polymerase chain reaction in the CSF has been described, but is only available in specialized laboratories [2]. As demonstrated in this case, false negative results are possible if serologic testing is restricted to single admission samples (impeding documented seroconversion). Paired serologic testing in weekly time intervals increases diagnostic detection as seroconversion can be rare: only 2 cases of documented seroconversion in human neuroangiostrongyliasis were identified in a systematic literature search $[12,15]$.

The course of disease is determined by the parasite's biology in the human host. Humans are considered dead-end hosts as the larvae cannot develop to adult worms and die after completed migration to the CNS. This explains the self-limiting course of disease $[3,5]$ and why most infections are mild. However, prolonged and severe courses as well as fatal cases have been reported $[16,17]$. Repeated lumbar punctures are an effective means to relieve headaches by reducing intracranial pressure [9]. Administration of steroids can reduce frequency of lumbar punctures, duration of headache and analgesics use [18]. Anthelmintic drugs are effective against early larval stages [19] and may reduce duration of headache [20]. However, their role in the treatment of neuroangiostrongyliasis remains controversial as they potentially exacerbate neurological symptoms via inducing host immune-inflammatory responses $[3,21]$. Consequently, the recommended treatment regimens for $A$. cantonensis are still debated. Some studies found that combinations of antihelminths and steroids are effective [22-24] or at least not inferior and safe [25] in the treatment of EOM caused by A. cantonensis. In our case, a combination of albendazole with prednisolone was effective and resulted in a complete recovery.

\section{Conclusion}

Ecological, biological and economic changes can exert their effects on the epidemiology of communicable diseases. Health care providers are increasingly confronted with "exotic" diseases in a merging and globalized world. Even though EOM is rare, preemptive treatment with subsequent follow-up serology is advised if neuroangiostrongyliasis is suspected. Early diagnosis and appropriate management results in favorable outcomes in the majority of cases.

\begin{abstract}
Abbreviations
BD: Twice per day; CBC: Complete blood count; CNS: Central nervous system; CSF: Cerebrospinal fluid; CT: Computed tomography; EITB: Enzyme-linked electroimmunotransfer blot; EOM: Eosinophilic meningitis; LP: Lumbar puncture; MRI: Magnetic resonance imaging; OD: Once per day; VFR: Visiting friends and relatives
\end{abstract}

\section{Acknowledgements}

We would like to express our appreciation to the patient presented in this manuscript for the cooperation and detailed information provided. We would like to thank Dr. Nobuaki Akao from the Department of Environmental Parasitology, Tokyo Medical and Dental University, Japan, for provision of the antigen.

Special thanks to Rosalie Zimmermann for collating and updating the map in Fig. 1, to Sven Poppert for his critical review and to Gaye Proctor for her diligent proofreading of the manuscript.

\section{Funding}

No funding was obtained.

Availability of data and materials

Not applicable

\section{Authors' contributions}

Conceptualization TB, JB. Case management SB, JB, TB, AN. Data curation SB, KA, TT, MTR, BN. Supervision DHP. Writing - original draft TB, SB. Writing, review, editing TB, SB, KA, TT, MTR, BN, DHP, AN, JB. All authors have read and approved the final version of this manuscript. The contributor's roles listed above follow the Contributor Roles Taxonomy (CRediT) managed by The Consortia Advancing Standards in Research Administration Information (CASRAI) (https://casrai.org/credit/).

Ethics approval and consent to participate

Not applicable

\section{Consent for publication}

Written informed consent was obtained from the patient described in this manuscript.

\section{Competing interests}

The authors declare that they do not have any competing interests.

\section{Publisher's Note}

Springer Nature remains neutral with regard to jurisdictional claims in published maps and institutional affiliations.

\section{Author details}

${ }^{1}$ Department of Medicine, Swiss Tropical and Public Health Institute, Basel, Switzerland. ${ }^{2}$ Faculty of Medicine, University of Basel, Basel, Switzerland. ${ }^{3}$ Shoklo Malaria Research Unit, Mahidol-Oxford Tropical Medicine Research Unit, Faculty of Tropical Medicine, Mahidol University, Mae Sot, Thailand. ${ }^{4}$ Centre for Tropical Medicine and Global Health, Nuffield Department of Medicine, University of Oxford, Old Road Campus, Oxford, UK. ${ }^{5}$ Department of Infectiology, Luzerner Kantonsspital, Luzern, Switzerland. ${ }^{6}$ Division of Hematology and Hematology Laboratory, Luzerner Kantonsspital, Luzern, Switzerland. ${ }^{7}$ Division of Nuclear Medicine and Radiology, Luzerner Kantonsspital, Luzern, Switzerland.

Received: 26 February 2019 Accepted: 8 April 2019

Published online: 15 April 2019

\section{References}

1. Carpio A, Romo ML, Parkhouse RME, Short B, Dua T. Parasitic diseases of the central nervous system: lessons for clinicians and policy makers. Expert Rev Neurother. 2016 Apr 2;16(4):401-14.

2. Barratt J, Chan D, Sandaradura I, Malik R, Spielman D, Lee R, et al. Angiostrongylus cantonensis: a review of its distribution, molecular biology and clinical significance as a human pathogen. Parasitology. 2016;143(9): 1087-118.

3. Wang Q-P, Lai D-H, Zhu X-Q, Chen X-G, Lun Z-R. Human angiostrongyliasis. Lancet Infect Dis. 2008 Oct;8(10):621-30. 
4. Kliks MM, Palumbo NE. Eosinophilic meningitis beyond the Pacific Basin: the global dispersal of a peridomestic zoonosis caused by Angiostrongylus cantonensis, the nematode lungworm of rats. Soc Sci Med. 1992 Jan;34(2): 199-212.

5. Ansdell V, Wattanagoon Y. Angiostrongylus cantonensis in travelers: clinical manifestations, diagnosis, and treatment. Curr Opin Infect Dis. 2018;31(5): 399-408.

6. Diaz JH. Global climate changes and international trade and travel: effects on human health outcomes is. In: Reference module in earth systems and environmental sciences [internet]. Elsevier; 2018 [cited 2018 Oct 13]. Available from: http://www.sciencedirect.com/science/article/pii/ B9780124095489109728.

7. Chang KH. Han MH. MRI of CNS parasitic diseases. J Magn Reson Imaging. 1998;8(2):297-307.

8. Kuberski T. Eosinophils in cerebrospinal fluid: criteria for eosinophilic meningitis. Hawaii Med J. 1981;40(4):97-8.

9. Graeff-Teixeira C, da Silva ACA, Yoshimura K. Update on eosinophilic meningoencephalitis and its clinical relevance. Clin Microbiol Rev. 2009; 22(2):322-48.

10. Tseng Y-T, Tsai H-C, Sy CL, Lee SS-J, Wann S-R, Wang Y-H, et al. Clinical manifestations of eosinophilic meningitis caused by Angiostrongylus cantonensis: 18 years' experience in a medical center in southern Taiwan. J Microbiol Immunol Infect. 2011;44(5):382-9.

11. Nguyen Y, Rossi B, Argy N, Baker C, Nickel B, Marti H, et al. Autochthonous case of eosinophilic meningitis caused by Angiostrongylus cantonensis, France, 2016. Emerg Infect Dis. 2017;23(6):1045-6.

12. Senanayake SN, Pryor DS, Walker J, Konecny P. First report of human angiostrongyliasis acquired in Sydney. Med J Aust. 2003;179(8):430-1.

13. Wang Q-P, Wu Z-D, Wei J, Owen RL, Lun Z-R. Human Angiostrongylus cantonensis: an update. Eur J Clin Microbiol Infect Dis. 2012 Apr;31 (4):389-95.

14. Murphy GS, Johnson S. Clinical aspects of eosinophilic meningitis and meningoencephalitis caused by Angiostrongylus cantonensis, the rat lungworm. Hawaii J Med Public Health. 2013;72(6 Suppl 2):35-40.

15. Espírito-Santo MCC. Do, pinto PLS, Mota DJG da, Gryschek RCB. The first case of Angiostrongylus cantonensis eosinophilic meningitis diagnosed in the city of São Paulo, Brazil. Rev Inst Med Trop Sao Paulo. 2013;55(2):129-32.

16. Chotmongkol V, Sawanyawisuth K. Clinical manifestations and outcome of patients with severe eosinophilic meningoencephalitis presumably caused by Angiostrongylus cantonensis. Southeast Asian J Trop Med Public Health. 2002;33(2):231-4.

17. Blair NF, Orr CF, Delaney AP, Herkes GK. Angiostrongylus meningoencephalitis: survival from minimally conscious state to rehabilitation. Med J Aust. 2013;198(8):440-2.

18. Sawanyawisuth K, Chotmongkol V. Chapter 15 - Eosinophilic meningitis. In: Garcia HH, Tanowitz HB, Del Brutto OH, editors. Handbook of Clinical Neurology [Internet]. Elsevier; 2013 [cited 2018 Oct 14]. p. 207-15. (Neuroparasitology and Tropical Neurology; vol. 114). Available from: http:// www.sciencedirect.com/science/article/pii/B9780444534903000157

19. Hwang KP, Chen ER. Larvicidal effect of albendazole against Angiostrongylus cantonensis in mice. Am J Trop Med Hyg. 1988:39(2):191-5.

20. Jitpimolmard S, Sawanyawisuth K, Morakote N, Vejjajiva A, Puntumetakul M, Sanchaisuriya K, et al. Albendazole therapy for eosinophilic meningitis caused by Angiostrongylus cantonensis. Parasitol Res. 2007;100(6):1293-6.

21. Prociv P, Turner M. Neuroangiostrongyliasis: the "subarachnoid phase" and its implications for anthelminthic therapy. Am J Trop Med Hyg. 2018;98(2): 353-9.

22. Chotmongkol V, Wongjitrat C, Sawadpanit K, Sawanyawisuth K. Treatment of eosinophilic meningitis with a combination of albendazole and corticosteroid. Southeast Asian J Trop Med Public Health. 2004;35(1):172-4.

23. Chotmongkol V, Sawadpanitch K, Sawanyawisuth K, Louhawilai S, Limpawattana P. Treatment of eosinophilic meningitis with a combination of prednisolone and mebendazole. Am J Trop Med Hyg. 2006;74(6):1122-4.

24. Diao Z, Wang J, Qi H, Li X, Zheng X, Yin C. Treatment of angiostrongyliasis using a combination of albendazole and dexamethasone: the results of a retrospective and comparative study. Ann Trop Med Parasitol. 2011;105(1):65-9.

25. Chotmongkol V, Kittimongkolma S, Niwattayakul K, Intapan PM, Thavornpitak Y. Comparison of prednisolone plus albendazole with prednisolone alone for treatment of patients with eosinophilic meningitis. Am J Trop Med Hyg. 2009;81(3):443-5.

Ready to submit your research? Choose BMC and benefit from:

- fast, convenient online submission

- thorough peer review by experienced researchers in your field

- rapid publication on acceptance

- support for research data, including large and complex data types

- gold Open Access which fosters wider collaboration and increased citations

- maximum visibility for your research: over $100 \mathrm{M}$ website views per year

At BMC, research is always in progress.

Learn more biomedcentral.com/submissions 